\title{
A new online tool for visualization of volumetric data
}

To the editor: New popular microscopy techniques (Structured Illumination, Selective Plane Illumination Microscopy (SPIM), and Optical Projection Tomography (OPT), for example) and many other biomedical imaging modalities (Magnetic Resonance Imaging (MRI), X-Ray Computed Tomography) generate an explosive amount of volumetric data. Techniques such as SPIM capture $>50 \mathrm{M}$ voxels per minute, generating tens of terabytes of data per sample [1]. It is now possible to image whole organs and even animals at single cell resolution to produce so called 'digital embryos' [2]. Whilst highly specialized software packages are available for the analysis and visualization of such data, their use requires expert skills and they are often expensive. Furthermore, their capacity to share data is limited and there are no means for interactive exploration of the data by third parties.

We wish to inform the readers of Nature Photonics that we have developed an easy-to-use and powerful open-source visualization tool that enables researchers to share their volumetric image data online. The tool, called First Person Bioimage (FPBioimage), allows third parties to view and interact with datasets in their entirety. It can be downloaded for free at our website http://fpb.ceb.cam.ac.uk. FPBioimage provides the viewer with an immersive experience for the exploration of complex three-dimensional data. It makes use of the latest graphics capabilities embedded in all modern web browsers, and therefore no separate software is required for viewing. The tool is fast and user responsive, requires no training for use, and includes advanced rendering and data manipulation capabilities. Data can be intuitively explored from a 'first-person perspective', akin to navigating virtual space in modern computer games, allowing users to conceptualize and contextualize details in the data to aid biological and physical interpretation (see Supplementary Video 1).

Figure 1 shows screenshots of datasets obtained with OPT [3], MRI [4], and light-sheet microscopy [2], demonstrating different rendering and data manipulation capabilities. Although the tool was originally conceived for use in conjunction with bioimaging data, it is suited for the visualisation of any volumetric data. Other examples shown in Supplementary Figures 1-8 include the volumetric visualization of atomic wavefunctions and 3D electric fields in photonic devices. We have also ported FPBioimage to the Android and iOS mobile platforms for viewing on handheld devices. For a truly immersive experience of the data the app supports a virtual reality mode for viewing with a VR headset (see Supplementary Video 2).

From a researcher's perspective the tool now makes it possible to easily share volumetric data globally, providing anyone full and interactive access to the data via a web browser. In future, we hope this changes the way research data are shared and suggest that publishers will use the software as a tool for online publication. Data can thus be shared in their entirety, moving on from the current practice of providing selective views or movies shown from a single perspective, which are not capable of providing a full understanding of the data.

\section{References:}

[1] Reynaud, E., Peychi, J., Huisken, J., Tomancak, P. Nat. Methods 12, 30-34 (2015).

[2] Tomer, R., Khairy, K., Amat, F., \& Keller, P. J. Nat. Methods, 9(7), 755-763 (2012).

[3] Sharpe, J., Ahlgren, U., Perry, P., Hill, B., et. al. Science, 296(5567), 541-545 (2002).

[4] Levoy, M. IEEE Comput. Graph. Appl. 8(3), 29-37 (1988).

Acknowledgements: We thank M. Mantle, P. Keller, R. Chhetri, J. Swoger, and J. McGinty for the provision of volumetric image data. This work was sponsored by the Engineering and Phyiscal Sciences Research Council (EPSRC) UK, the Medical Research Council (MRC) UK and the Wellcome Trust.

\section{Marcus Fantham and Clemens F. Kaminski*}

Department of Chemical Engineering and Biotechnology, University of Cambridge

*e-mail: cfk23@cam.ac.uk 

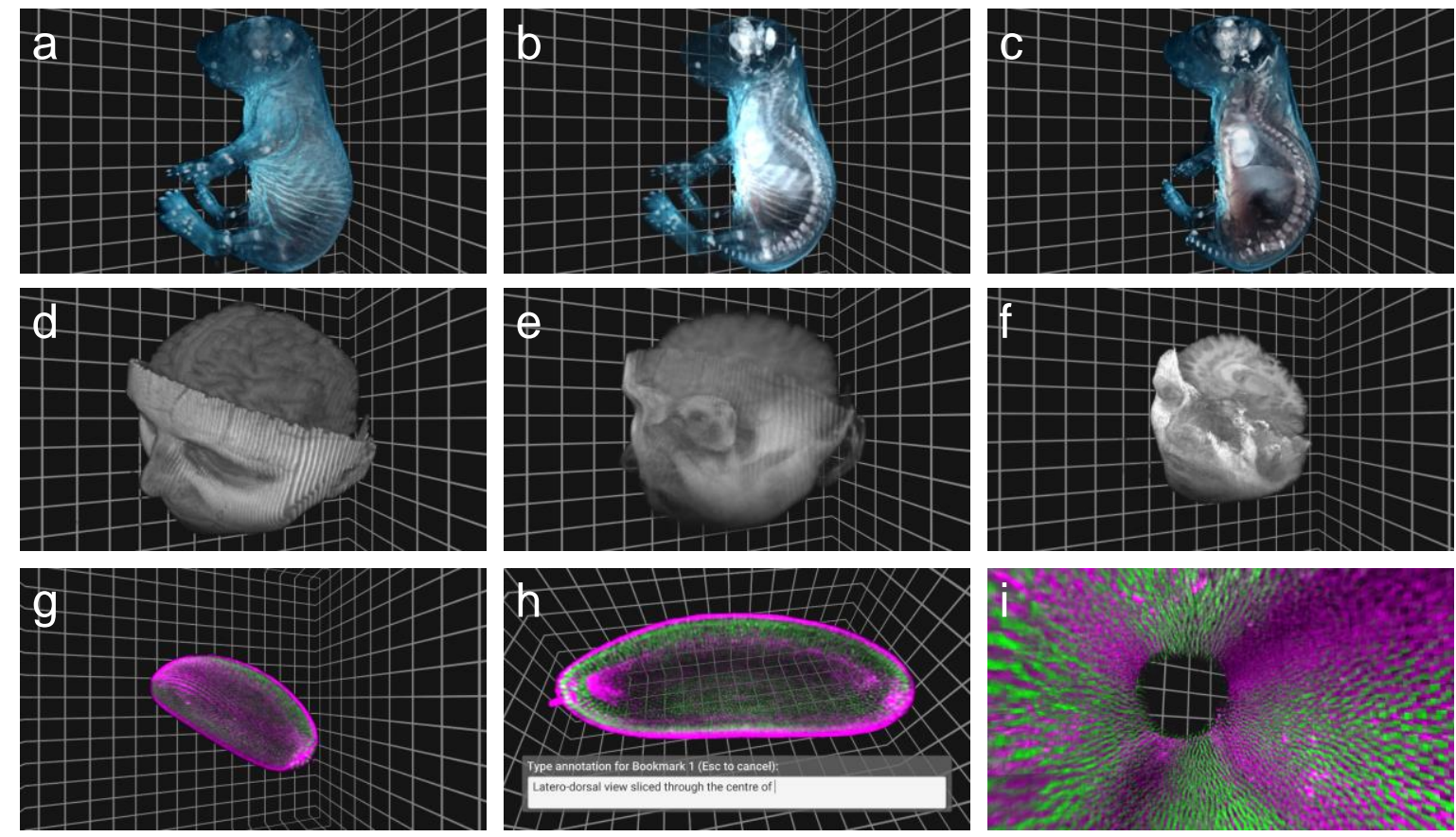

Figure 1: Screenshots of various example data demonstrating different rendering options of FPBioimage. (a-c) 4-colour OPT data of a mouse embryo; (d-f) MRI data of a human head; and (g-i) 2-colour light-sheet data of a drosophila embryo. The viewer's built-in transparency feature has been used to render (b) and (e), revealing data within the volume; the cutting tool has been used for (c), (f), (h) and (i), removing outer voxels from the data; and (h) shows a bookmark in creation, which can be shared with other users. Raw data provided by J. McGinty (a-c) [3], Stanford Volume Data Archive (d-f) [4], and P. Keller (g-i) [2]. 